\title{
Notes on the slow dynamics in dilute lyotropic lamellar phase
}

\author{
Shuji Fujii*, Yuji Sasaki, Hiroshi Orihara \\ Faculty of Engineering, Division of Applied Physics, Hokkaido University, N13W8, Sapporo, Hokkaido 060-8628, Japan
}

Received: 26 October 2018 / Accepted: 6 November 2018

(C) Japanese Society of Biorheology 2019

\begin{abstract}
In this study, we try to extract the dynamics of the dislocations by measuring the time auto-correlation function of the scattered light intensity in the dilute lamellar phase. Our finding is that the lyotropic lamellar phase shows a bimodal decay of the correlation function. Fitting with the dispersion relation for the undulation fluctuation indicates that the fast mode is attributed to the undulation fluctuation of bilayer membranes. On the other hand, the slow relaxation mode is not explained by the undulation fluctuation. We interpret that the slow mode would be originated from a climb motion of the dislocation. Bimodal relaxation process would be attributed to a heterogeneous distribution of the dislocations in the lamellar structure.
\end{abstract}

Keywords lamellar phase, defect, slow dynamics, dynamic light scattering

\section{Introduction}

Defect-mediated phenomena are a fascinating subject in soft matter science [1]. Defect plays an important roles in macroscopic properties such as phase transitions and rheology of liquid crystalline systems [2-6]. Phase behavior and rheology of layered systems are also closely correlated with the dynamics of dislocation. For example, smectic-nematic transition is known to be induced by melting of the layered structure. Melting of the smectic phase is induced by a proliferation of the dislocations $[7,8]$. Indeed, the proliferation of the dislocations was experimentally confirmed in the vicinity of the phase transition point [8]. Regarding the rheology of the layered structures, the shear modulus is well scaled by the effective interfacial tension of the defects $[4,9-11]$. Even the shear-thinning behavior of the layered systems is dominated by production-annihilation processes of the dislocations $[9,12-14]$. In addition, nonequilibrium

*E-mail: sfujii@eng.hokudai.ac.jp phase transition phenomena such as shear-induced structural and orientational transitions are also significantly affected by a dynamic rearrangement of the dislocations [15].

Even though all the above reports suggests the importance of the dislocations, there is no compelling observational evidence of the dislocation dynamics. Dynamics of the lamellar phase has been studied by using a dynamic light scattering (DLS) technique [16-18]. However, those studies were performed under strong confinement in order to obtain well aligned lamellae without the dislocations.

In this brief communication, we perform the dynamic light scattering measurements of the dilute lamellar phase without the spatial confinement. We show the presence of fast and slow relaxation modes in the dilute lamellar phase. The fast mode is associated with the undulation fluctuation of the membranes. The slow mode showing a very slow relaxation time, $\sim 10 \mathrm{~s}$, is interpreted as being the consequent of a climb motion of the dislocations induced by the undulation fluctuation. Slight increase of the surfactant concentration makes the DLS measurements difficult because of this slow dynamics.

\section{Experimental}

We used nonionic surfactant, tri-ethylene glycol monodecylether $\left(\mathrm{C}_{10} \mathrm{E}_{3}\right)$ purchased from Nikko chemicals Co., Ltd. Dilute lamellar phase was prepared by dissolving $\mathrm{C}_{10} \mathrm{E}_{3}$ into the distilled water with a weight fraction of $2 \%$. Membrane thickness $\delta$ and intermembrane spacing $d$ at 2 wt. $\%$ are 2.76 and $140 \mathrm{~nm}$, respectively. Here, the intermembrane spacing $d$ of the dilute lamellar phase is almost inversely proportional to the surfactant weight fraction $\simeq \delta / d$. The density of $\mathrm{C}_{10} \mathrm{E}_{3}$ is $0.938 \mathrm{~g} / \mathrm{cm}^{3}$. Dynamic light scattering (DLS) measurements were performed using home-made set up equipped with a parallel plate shear flow cell. In this setup, the incident light, shear cell, and detector were vertically aligned by following a previous report [19]. 
Figure 1 describes the geometrical alignment of the shear cell and scattering angle. Sample thickness was $1 \mathrm{~mm}$, which is much thicker than the sample cell used in conventional DLS measurements of the dilute lamellar phase. The polarization of the incident light (He-Ne laser with $\lambda=632.8 \mathrm{~nm}$ ) and the scattered light were both vertical to the plane in order to observe the concentration fluctuation. The wave vector $\mathbf{q}=\left(q_{x}, q_{y}, q_{z}\right)$ is defined by the angle between the incident laser and the detector. In our setup, three components are described as $q_{x}=q \cos (\theta-\phi / 2)$, $q_{y}=0, q_{z}=q \sin (\theta-\phi / 2)$, with $q=(4 \pi n / \lambda) \sin (\phi / 2)$, respectively. Here, angle $\theta$ controls the orientation of the lamellae against the scattering vector, and $\phi$ controls the modulus of the wave vector. In this study, the angle $\theta$ was fixed at $12^{\circ}$ and $\phi$ was scanned from 10 to $35^{\circ}$. $n$ is a refractive index of solution. Here, we assumed $n$ to be the same as the refractive index of the solvent. The light scattered from the sample was detected with an avalanche photo diode, SPCM- AQR-14-FC (PerkinElmer, Massachusetts, USA) in single-photon counting mode and processed by a digital photon counter Flex03-01 (correlator.com, New Jersey, USA). All measurements were performed at $T=25^{\circ} \mathrm{C}$. Before measurements, sample solution was always subjected to the shear flow of $10 \mathrm{~s}^{-1}$ for $10 \mathrm{~min}$ in order to adjust the initial condition. Time auto-correlation function was measured for $60 \mathrm{~min}$ after the cessation of shear. The same procedure was repeated 10 times at the same experimental condition.

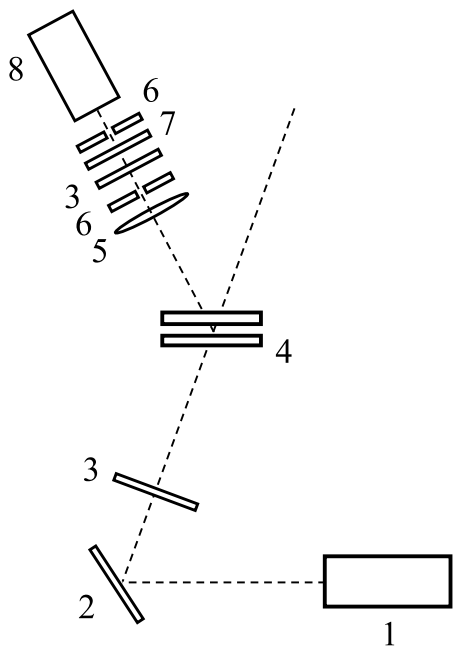

(a)

\section{Results and Discussion}

Figure 2 shows time auto-correlation functions $g_{1}(\tau)$ obtained for the dilute lamellar phase. Different symbols correspond to the data from each trial at the same experimental condition.

For the perfectly aligned lamellar phase under strong

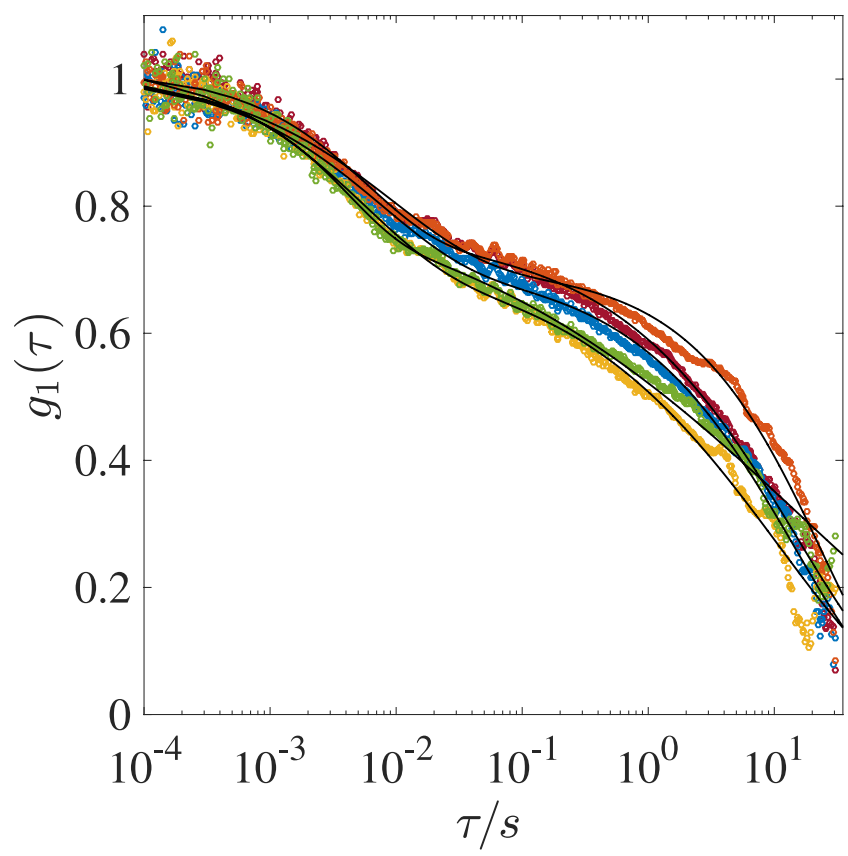

Fig. 2 Time auto-correlation function of the dilute lamellar phase at $\phi=25^{\circ}$ at $T=25^{\circ} \mathrm{C} \cdot g_{1}(\tau)$ obtained at the same experimental conditions are compared. Solid curves are the best fit to the double stretched exponential function eq. 1 .

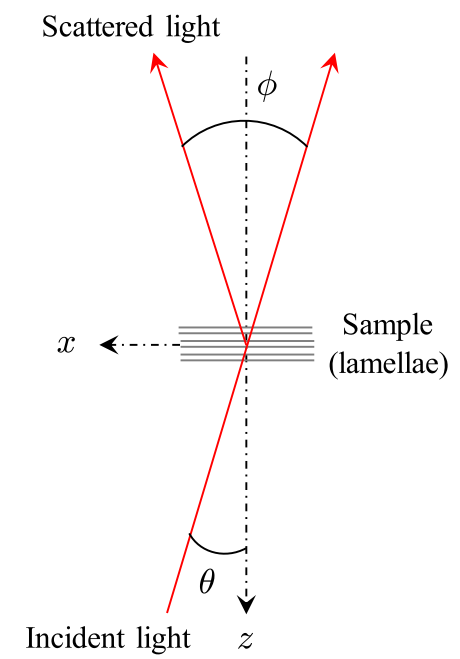

(b)

Fig. 1 Setup of the vertically mounted DLS. (a): A schematic drawing of the vertical small angle DLS setup: (1) He-Ne laser, (2) mirror, (3) polarizers, (4) sample (parallel plate shear cell), (5) achromatic lens, (6) pinholes, (7) bandpass filter, (8) avalanche photodiode (APD). (b): Geometry around the sample cell. Angle $\theta$ controls the orientation of the lamellae against the scattering vector. Angle $\phi$ was scanned from 10 to $35^{\circ}$, while $\theta$ was fixed at $12^{\circ}$. 
confinement, only single relaxation behavior originated from the undulation fluctuation is expected as previously reported [16-18]. We find, however, that the correlation function always appears to be bimodal, which gives us two characteristic decay rates with different time scales. We just note that this bimodal relaxation is also observed even without shear treatment. For all the measurements, we can detect the fast relaxation behavior with a good reproducibility, while the slow mode deviates in every measurements.

In order to characterize each decay mode, we analyze the correlation functions by using a double stretched exponential function given as

$g_{1}(\tau)=A \exp \left[-\left(\Gamma_{\mathrm{f}} t\right)^{\beta_{\mathrm{f}}}\right]+(1-A) \exp \left[-\left(\Gamma_{\mathrm{s}} t\right)^{\beta_{\mathrm{s}}}\right]$

where $\Gamma_{\mathrm{f}}$ and $\Gamma_{\mathrm{s}}$ are the fast and slow decay rates, respectively. $\beta_{\mathrm{f}}$ and $\beta_{\mathrm{s}}$ are stretched exponent values for each decay rate. And $A$ is an amplitude of the fast mode. As shown in Fig. 2, eq. 1 well fits to the experimental data. Averaged relaxation times estimated as the reciprocal of the decay rate, $\tau=1 / \Gamma$, are $\left\langle\tau_{\mathrm{f}}\right\rangle=5.9 \times 10^{-3} \mathrm{~s}$ and $\left\langle\tau_{\mathrm{s}}\right\rangle=20 \mathrm{~s}$, respectively. And the standard deviation of the relaxation time corresponding to each mode are $1.6 \times 10^{-3}$ and 9.6, respectively. High standard deviation of the slow mode suggests that the slow dynamics is scattered over wide range in every measurements. Poor reproducibility of the slow relaxation behavior indicates an inhomogeneous nature of the dynamics of the lamellar phase. The slow mode appears to depend on location where the incident laser light is irradiated. Similar behavior, high standard deviation of the slow modes, is also observed in the correlation function obtained at different scattering angles $\phi$ (data not shown).

We estimated the averaged value of the stretched exponent values for the fast and slow modes to be $\left\langle\beta_{\mathrm{f}}\right\rangle=0.79$ and $\left\langle\beta_{\mathrm{s}}\right\rangle=0.45$, respectively. The standard deviations of each stretched exponent value are 0.03 and 0.15 , respectively. Low stretched exponential value in the slow mode indicates a polydispersity of the relaxation time. We note that the bimodal behavior is also detected in different surfactant concentrations of 4, 6, and 8 wt.\%. However, in those correlation function measurements at higher concentration ( $i 2$ wt.\%), the slow mode is not nicely obtained because of insufficient integration time of the correlation function. Slight increase of the surfactant concentration causes a remarkable increase of the relaxation time of the slow mode. For measuring the correlation function with such slow dynamics, we need a different setup such as an image correlation spectroscopy with CCD camera, which allows the measurement of the slow dynamics even in colloidal glass systems.

As previous reports show, the relaxation process of the well aligned lamellar phase under spatial confinement is primary due to the undulation fluctuation of the membranes $[16,17]$. Membrane displacement fluctuation associated with the concentration fluctuations is governed by the elastic restoring force and dissipation. The characteristic decay rate corresponding to the undulation fluctuation coupled to the concentration fluctuation is given by the dispersion relation

$\Gamma_{\mathrm{u}}=\frac{B q_{\mathrm{z}}^{2}+K q_{\mathrm{x}}^{4}}{\eta_{\mathrm{s}} q^{4}+q_{z}^{2} / \mu} q_{\mathrm{x}}^{2}$

where $B$ and $K$ are the smectic compression modulus and the bending modulus of the layers, and $\eta_{\mathrm{s}}$ and $\mu$ are dissipative parameters, the solvent viscosity and surfactant mobility, respectively. The surfactant mobility $\mu$ is found equal to $\mu=(d-\delta)^{2} / 12 \eta_{\mathrm{s}}$ [20]. In this equation, $q_{\mathrm{z}}$ and $q_{\mathrm{x}}$ are the projections of the wave vector $\mathbf{q}$ along the stacking direction $z$ and in the plane of the lamellae $x$, respectively. Thus, each wave vector is related by $q^{2}=q_{\mathrm{z}}^{2}+q_{\mathrm{x}}^{2}$.

Figure 3 shows the fast and slow decay rates as a function of the square of the scattering vector. Within experimental errors, the data is compatible with a linear dependence passing through the origin. Solid line in panel (a) is a fitting curve obtained using eq. 2 with $B$ and $K$ as fitting parameters. Obtained values for $B=0.5 \mathrm{~Pa}$ and $K=7.65 \times 10^{-15} \mathrm{~N}$ almost agree with the previous report on the DLS measurement of the confined dilute lamellar phase of nonionic $\mathrm{C}_{12} \mathrm{E}_{5} / \mathrm{H}_{2} \mathrm{O}$ system [16]. This consistency suggests that the fast mode can be attributed to the undulation
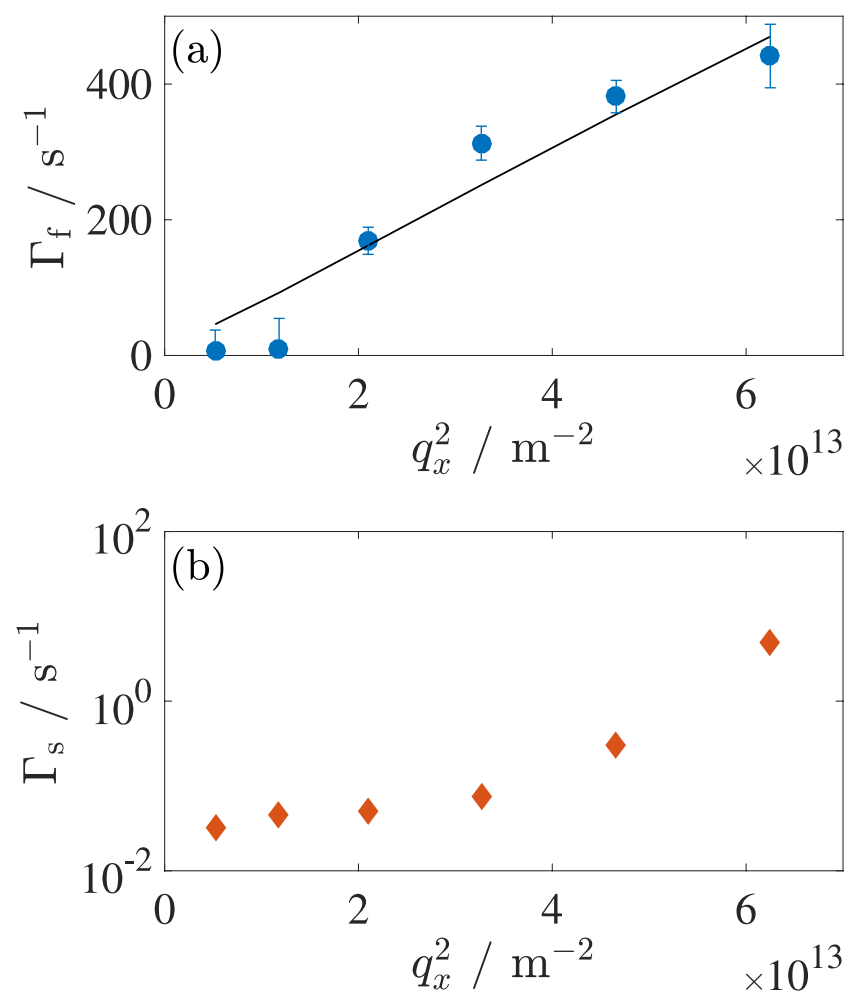

Fig. 3 In panel (a), the decay rate of the fast mode $\Gamma_{\mathrm{f}}$ is plotted as a function of $q_{\mathrm{x}}^{2}$. Solid line is the best fit to eq. 2. In panel (b), the decay rate of the slow mode $\Gamma_{\mathrm{s}}$ was plotted as a function of $q_{\mathrm{x}}^{2}$ in semilogarithmic graph. 
fluctuation. Low standard deviation in the decay rate of the fast mode is a consequence of a homogeneous fluctuation dynamics without a local anomaly. Therefore, we conclude that the fast mode corresponds to the undulation fluctuation. Under the spatial confinement effect, the slow mode will be eliminated and single relaxation behavior is expected as previous reports detected. However, we could not reduce the sample thickness, because of the geometrical problem of our experimental setup.

Appearance of the slow relaxation mode indicates a presence of additional dynamics of the lamellar phase, which does not present in the well aligned lamellar phase. Here we would like to consider the origin of the slow mode. As we mentioned, poor reproducibility of the slow mode suggests that the relaxation behavior depends on the location of measurement points in the sample solution. Such local anomaly indicates inhomegeneous structure of the lamellar phase. One plausible reason for the inhomogeneity would be attributed to the presence of dislocations in the lamellar alignment $[2,21]$. Dislocations in the lamellar phase distribute inhomogeneously [5]. At low surfactant concentration of 2 wt. $\%$, nearest neighbor distance between in-plane dislocations can be a magnitude of the order of $100 \mu \mathrm{m}$, which is roughly comparable to the size of the incident beam. In such length scale of the nearest neighbor distance between dislocations, the measurement of the time auto-correlation function will be significantly affected by the local density of the dislocations.

Dislocations behave as elastic strings [5, 6, 22]. If dislocations form a network, elastic force balance stabilizes the dislocation array and results in very slow process. One characteristic motion of the dislocation is a climb motion, which is induced by compressive or dilatational stress [2]. Under the action of the compressive or dilatational stress in the normal direction of the membranes, the dislocation moves in-plane direction. It is known that the wedge cell creates the dislocation in the middle of the lamellar solution $[2,3]$. Therefore, the characteristic time of this motion is obtained by observing the movement of the dislocation under compression of the wedge cell. Oswald et al. measured the characteristic time of the climb motion in nonionic surfactant $\mathrm{C}_{12} \mathrm{E}_{5} / \mathrm{H}_{2} \mathrm{O}$ lamellar phase by applying compressive strain [23]. Obtained characteristic time of the climb motion was approximately $10^{\circ} \mathrm{s}$. They also theoretically described the characteristic time of the climb motion in wedge cell as,

$\tau_{\mathrm{d}}=\frac{L}{B m \alpha}$,

where $L, m$, and $\alpha$ are the characteristic size of the lamellae, mobility of the dislocation, and angle of the wedge cell, respectively. They confirmed the above relation well describes the characteristic time of the climb motion. Another interesting finding is that the characteristic time becomes slower as the wedge angle approaches to parallel.
In the lamellar phase confined in the wedge cell, the angle of the wedge cell is approximately the same as a local inclination angle between two neighboring membranes upper and lower the edge dislocation. If we assume that the angle $\alpha$ can be regarded as the local inclination angle of the membranes, eq. 3 is applicable to the dynamics of the dislocation of the lamellar phase even in the parallel plate. The inclination angle of the membranes around the dislocation $\alpha$ can be roughly $0.1 \mathrm{rad}$ [3]. In this assumption, the characteristic size $L$ would be regarded as the distance between two neighboring membranes around the dislocation, which is the magnitude of the order of $100 \mathrm{~nm}$. The characteristic time of the climb motion $\tau_{\mathrm{d}}$ is thus estimated to be $\sim 10 \mathrm{~s}$ with a typical value of the mobility of the dislocation, $10^{-7} \mathrm{~m}^{3} \mathrm{~N}^{-1} \mathrm{~s}^{-1}$. Estimated time is almost consistent with the relaxation time of the slow mode. To drive the climb motion, the force in the normal direction of the membranes is necessary. The driving force of the climb motion in the local scale would be a weak compressibility yielded by the undulation fluctuation. Compressive force in the normal direction of the membranes generated by the undulation fluctuation will drive the climb motion. To verify the validity of the climb motion, we should incline the shear cell intentionally to confirm the angle dependence of the slow relaxation mode, as eq. 3 suggests. Although we have not done such measurements because of the difficulty in the experimental setup, we interpret that the slow mode is attributed to the climb motion of the dislocations.

Another possibility on the origin of the slow mode might be the viscoelastic effect. The slow mode in the wormlike micellar systems is generated as a result of the collective diffusion and longitudinal stress relaxation mode [24, 25]. In such entangled systems, the viscoelastic effect can be attributed to the osmotic and shear moduli. In the dilute lamellar phase, however, these moduli are very small and almost negligible [26]. Actually, as the compression modulus $B$ is small in this study, the osmotic contribution, i.e., viscoelastic effect will not be the origin of the slow mode in the dilute lamellar phase.

For more systematic study of the dynamics of the dislocation, we need to perform statistical analysis on the slow mode. One possible way would be to follow the analytical method for gels with spatial inhomogeneity of the polymer network structure [27]. Dividing static and dynamic fluctuations by following their method, the dynamics of the lamellar phase with spatial inhomogeneities can be investigated in more detail.

\section{Summary}

We have focused on the dynamics of the dislocations within the dilute lyotropic lamellar phase. In the measurement of the time auto-correlation function, two relaxation modes were observed. Fast mode, which can be obtained 
with a good reproducibility, is attributed to the undulation fluctuation of the lamellar membranes by comparing the dispersion relation of the undulation fluctuation. On the other hand, the reproducibility of the slow mode was poor. And the characteristic time of the slow mode was much slower than the undulation fluctuation of the lamellar membranes. We interpret that the origin of the slow mode would be attributed to the climb motion of the dislocations in the lamellar structure.

The presence of the dislocations does not change the structure of the undulation fluctuation mode but induce the second relaxation process, which is polydispersive and much slower than the undulation fluctuation. Undulation fluctuation still follows the same relation as eq. 2 . When the dislocations are present in the lamellar phase, we observe bimodal relaxation process. The slow relaxation process is much slower than the undulation fluctuation. Although we have not identified the second mode yet, we presume that it corresponds to the climb motion of the dislocations, which is induced by compressibility due to the undulation fluctuation of the membranes.

Acknowledgements This work was supported by JSPS KAKENHI Grant-in-Aid for Scientific Research on Innovative Areas JP $18 \mathrm{H} 04469$.

\section{References}

1. Larson RG. The Structure and Rheology of Complex Fluids. Oxford University Press, Oxford and New York; 1999.

2. Oswald P, Pieranski P. Smectic and Columner Liquid Crystals. Taylor \& Francis, Boca Raton; 2006.

3. Kleman M, Lavrentovich OD. Soft Matter Physics: An Introduction. Springer; 2003.

4. Horn RG, Kleman M. Observations on shear-induced textures and rheology of a smectic-A phase. Ann Phys. 1978; 3: 229.

5. Basappa G, Suneel, Kumaran V, Nott PR, Ramaswamy S, Naik VM, Rout D. Structure and rheology of the defect-gel states of pure and particle-dispersed lyotropic lamellar phases. Eur Phys J B. 1999; 12: 269.

6. Ramos L, Zapotocky M, Lubensky TC, Weitz DA. Rheology of defect networks in cholesteric liquid crystals. Phys Rev E. 2002; 66: 031711 .

7. Helfrich W. Defect model of the smectic A-nematic phase transition. J Phys (Paris). 1978; 39: 1199.
8. Moreau P, Navailles L, Giermanska-Kahn J, Mondain-Monval O, Nallet F, Roux D. Dislocation- loop-mediated smectic melting. Europhys Lett. 2006; 73: 49.

9. Fujii S, Ishii Y, Komura S, Lu C-Y D. Smectic rheology close to the smectic-nematic transition. Europhys Lett. 2010; 90: 64001.

10. Fujii S, Komura S, Ishii Y, Lu C-Y D. Elasticity of smectic phase with focal conic domains. J Phys Cond Matt. 2011; 23: 235105.

11. Fujii S, Komura S, Lu C-Y D. Structural rheology of smectic A phase. Materials. 2014; $7: 5146$.

12. Meyer C, Asnacios S, Bourgaux C, Kleman M. Rheology of lyotropic and thermotropic lamellar phase. Rheol Acta. 2000; 39: 223.

13. Meyer C, Asnacios S, Kleman M. Universal properties of lamellar systems under weak shear. Eur Phys J E. 2001; 6: 245.

14. Lu C-Y D, Chen P, Ishii Y, Komura S, Kato T. Non-linear rheology of lamellar liquid crystals. Eur Phys J E. 2008; 25: 91.

15. Fujii S, Yamamoto Y. Dynamic orientation transition of the lyotropic lamellar phase at high shear rates. Soft Matter. 2015; 11: 9330.

16. Oizumi J, Kimura Y, Ito K, Hayakawa R. Dynamic light scattering of a lyotropic piquid crystal lamellar phase and a sponge phase. Mol Cryst Liq Cryst. 1997; 303: 63.

17. Freyssingeas E, Roux D, Nallet F. Quasi-elastic light scattering study of highly swollen lamellar and "sponge" phases. J Phys II France. 1997; 7: 913.

18. Nallet F, Roux D, Prost J. Dynamic light scattering study of dilute lamellar phases. Phys Rev Lett. 1989; 62: 276.

19. Kang K, Dhont JKG. Electric-field induced transitions in suspensions of charged colloidal rods. Soft Matter. 2010; 6: 273.

20. Brochard SF, de Gennes PG. Phase transition and turbulence: an introduction. Pramana Suppl. 1975; 1: 1.

21. Boltenhagen P, Lavrentvich O, Kleman M. Oily streaks and focal conic domains in $L_{\alpha}$ lyotropic liquid crystals. J Phys II France. 1991; 1: 1233.

22. Iwashita Y, Tanaka H. Pattern evolution of an edge-dislocation array in a lyotropic lamellar phase confined in a wedge-shaped cell: defect formation, relaxation, and recombination. Phys Rev E. 2008; 77: 041706.

23. Oswald P, Allain M. Influence of structural defects on the viscoelastic properties of a lamellar lyotropic phase. J Physique. 1985; 46: 831

24. Thuresson K, Nilsson S, Kjøniksen AL, Walderhaug H, Lindman B, Nyström B. Dynamics and rheology in aqueous solutions of associating diblock and triblock copolymers of the same type. J Phys Chem B. 1999; 103: 1425.

25. Li J, Ngai T, Wu C. The slow relaxation mode: from solutions to gel networks. Polymer J. 2010; 42: 609.

26. Jörgensen HB, Olsson U. Experimental study of undulation forces in a nonionic lamellar phase. Langmuir. 1996; 12: 4057.

27. Shibayama M, Fujikawa Y, Nomura S. Dynamic light scattering study of poly ( $N$-isopropylacrylamide-co-acrylic acid) gels. Macromolecules. 1996; 29: 6535. 